Review

\title{
Nutritional Support in Cancer Patients: A Position Paper from the Italian Society of Medical Oncology (AIOM) and the Italian Society of Artificial Nutrition and Metabolism (SINPE)
}

Riccardo Caccialanza ${ }^{\boxplus}$, Paolo Pedrazzoli², Emanuele Cereda ${ }^{1}$, Cecilia Gavazzi ${ }^{3}$, Carmine Pinto ${ }^{4}$, Agostino Paccagnella ${ }^{5}$, Giordano Domenico Beretta ${ }^{6}$, Mariateresa Nardi ${ }^{7}$, Alessandro Laviano ${ }^{8}$ and Vittorina Zagonel $^{9}$

1. Nutrition and Dietetics Service, Fondazione IRCCS Policlinico San Matteo, Pavia, Italy;

2. Division of Medical Oncology, Department of Hemato-Oncology Fondazione IRCCS Policlinico San Matteo, Pavia, Italy;

3. Clinical Nutrition Unit, National Cancer Institute, Milan, Italy;

4. Medical Oncology, IRCCS-Arcispedale S. Maria Nuova, Reggio Emilia, Italy;

5. Endocrinology, Metabolism and Nutrition Unit, Local Health Authority ULSS 9, Treviso, Italy;

6. Medical Oncology Unit, Humanitas Gavazzeni, Bergamo, Italy;

7. Clinical Nutrition Service, Veneto Institute of Oncology-IRCCS, Padova, Italy;

8. Department of Clinical Medicine, University La Sapienza, Rome, Italy;

9. Medical Oncology 1, Veneto Institute of Oncology-IRCCS, Padova, Italy.

$\square$ Corresponding author: Dr. Riccardo Caccialanza, Nutrition and Dietetics Service, Fondazione IRCCS Policlinico San Matteo, Viale Golgi 19 , 27100 Pavia, Italy. Tel.: +390382 501615; fax: + 390382 502801. E-mail: r.caccialanza@smatteo.pv.it

(C) Ivyspring International Publisher. Reproduction is permitted for personal, noncommercial use, provided that the article is in whole, unmodified, and properly cited. See http://ivyspring.com/terms for terms and conditions.

Received: 2015.09.11; Accepted: 2015.11.01; Published: 2016.01.01

\begin{abstract}
Malnutrition is a frequent problem in cancer patients, which leads to prolonged hospitalization, a higher degree of treatment-related toxicity, reduced response to cancer treatment, impaired quality of life and a worse overall prognosis. The attitude towards this issue varies considerably and many malnourished patients receive inadequate nutritional support.

We reviewed available data present in the literature, together with the guidelines issued by scientific societies and health authorities, on the nutritional management of patients with cancer, in order to make suitable and concise practical recommendations for appropriate nutritional support in this patient population. Evidence from the literature suggests that nutritional screening should be performed using validated tools (the Nutritional Risk Screening 2002 [NRS 2002], the Malnutrition Universal Screening Tool [MUST], the Malnutrition Screening Tool [MST] and the Mini Nutritional Assessment [MNA]), both at diagnosis and at regular time points during the course of disease according to tumor type, stage and treatment. Patients at nutritional risk should be promptly referred for comprehensive nutritional assessment and support to clinical nutrition services or medical personnel with documented skills in clinical nutrition, specifically for cancer patients. Nutritional intervention should be actively managed and targeted for each patient; it should comprise personalized dietary counseling and/or artificial nutrition according to spontaneous food intake, tolerance and effectiveness. Nutritional support may be integrated into palliative care programs. "Alternative hypocaloric anti-cancer diets" (e.g. macrobiotic or vegan diets) should not be recommended as they may worsen nutritional status.

Well-designed clinical trials are needed to further our knowledge of the nutritional support required in different care settings for cancer patients.
\end{abstract}

Key words: malnutrition, cancer, nutritional support 


\section{Introduction}

Malnutrition is a frequent problem in cancer patients, whose prevalence and degree mainly depend on tumor stage and site (1). Its negative consequences are prolonged hospitalization, a higher degree of treatment-related toxicity, reduced response to cancer treatment, lower activity level, impaired quality of life and a worse overall prognosis (2). Even minimal weight loss during chemo/radiotherapy (CRT) is associated with significantly reduced survival (3).

Nutritional support is a step by step intervention, which should be actively managed and targeted for each patient according to nutritional conditions, clinical status, planned oncologic treatment and expected outcome. Its goal is preventing or treating malnutrition, in order to allow the successful completion of oncologic treatments, improve prognosis and preserve functional status and quality of life $(4,5)$.

Although recommendations on the optimal management of nutritional support for patients with malignancies have been provided $(4,6,7)$, the attitude towards this issue varies considerably among oncologists, sometimes even within one center, and an important proportion of malnourished patients is reported not to receive adequate nutritional support (1). This could be related to the continuing insufficient awareness of nutritional problems among health care professionals (8), the lack of structured collaboration between oncologists and clinical nutrition specialists and the still limited number of clinical trials aimed at improving our understanding of the nutritional support required in different care settings for cancer patients.

Another worrying issue, which may hamper the appropriate nutritional care of cancer patients, is the expanding market of "alternative anti-cancer diets", which are not supported by scientific evidence and may lead to insufficient protein-calorie intake.

The purpose of this paper is to highlight the nutritional issues in cancer patients, thus allowing the Italian Association of Medical Oncology (AIOM) and the Italian Society of Artificial Nutrition and Metabolism (SINPE) to make suitable and concise practical recommendations for appropriate nutritional support in this patients' population.

We reviewed the available literature - prioritizing meta-analyses, systematic reviews and randomized controlled trials where available - and international guidelines on the nutritional management of patients with cancer. In addition, experts from the two societies, who are listed among the authors, provided additional clinical information which helped in clarifying some issues.

\section{Nutritional screening and assessment}

Early recognition of nutritional problems is the first key point for appropriate nutritional management of cancer patients. Different tools for nutritional screening have been validated in the oncologic setting and effectively allow the identification of patients at nutritional risk, who are likely to benefit from nutritional support. They are: the Nutritional Risk Screening 2002 (NRS 2002), the Malnutrition Universal Screening Tool (MUST), the Malnutrition Screening Tool (MST) and the Mini Nutritional Assessment (MNA) (9).

Nutritional screening should be performed using a validated tool upon diagnosis and systematically repeated at regular time points during the course of disease in patients with cancer type, stage or treatment potentially affecting nutritional status.

Patients at nutritional risk should be promptly referred for comprehensive nutritional assessment and support to clinical nutrition services or medical personnel with documented skills in clinical nutrition, specifically for cancer patients.

Recent studies have highlighted the importance of assessing body composition in cancer patients (10). Bioelectrical impedance vectorial analysis (BIVA) can be performed in different clinical settings and allow the suitable assessment of patients in whom calculation of body composition fails due to altered hydration (11). In particular, the primary output measure of this technique, phase angle, was found to be associated with functional status (12) and energy intake (13), and to be predictive of quality of life and prognosis in cancer patients $(12,14)$. As nutritional therapy is primarily intended to preserve or restore lean body mass, the assessment of body composition by BIVA should be integrated in the nutritional assessment of cancer patients.

\section{Indications for nutritional support}

Indications for nutritional support in cancer patients vary throughout the continuum of care, depending on whether patients are undergoing active oncologic treatment, are in remission or in a palliative stage. This means that regular nutritional monitoring is mandatory in all patients with cancer type, stage or treatment potentially affecting nutritional status.

Nutritional interventions should compensate for inadequate energy intake with the objective of improving clinical outcomes $(4,6,7,15)$.

The correct identification of candidates for nutritional support relies on the evaluation of current and expected nutritional status and energy intake. Accordingly, nutritional support should be provided to malnourished patients and those at nutritional risk, 
in particular when oral energy intake is already insufficient or expected to be inadequate $(<60 \%$ of estimated caloric requirements) for more than 7 days (4, $6,7,15)$.

Undernourished cancer patients with planned elective surgery should receive at least 7-day pre-operative nutritional support to improve post-operative outcomes, even if this may delay surgery (16).

Dietary counseling, including the use of oral nutritional supplements (ONS), should be the first-step towards achieving satisfactory energy intake. In presence of normal gut function and inadequate food intake, total or integrative enteral tube feeding must be considered. If enteral nutrition (EN) is not feasible due to gut dysfunction, symptoms which could be worsened by enteral support (i.e. nausea, vomiting, diarrhea) or patients' refusal, parenteral nutrition $(\mathrm{PN})$ is required for delivering nutritional support $(4,6,7,15)$.

\section{Nutritional counseling, complementary nutrients and oral nutritional supple- ments}

Nutritional counseling is the first-line of treatment in malnourished cancer patients or in those at nutritional risk, due to its proven efficacy in increasing protein-calorie intake, body weight and improving body composition $(17,18)$. In head and neck cancer patients undergoing CRT, nutritional counseling was found to be associated with lower CRT toxicity and symptom-induced morbidity (19), and to have beneficial effects on quality of life (20). Individualized nutrition intervention was also shown to improve survival in colorectal cancer patients (21).

Therefore, all malnourished or at nutritional risk cancer patients who are able to eat should be referred to a dietitian with documented skills in cancer patient care for appropriate dietary intervention and its monitoring. While taking into account individual preferences, ethnicity and culture, the optimization of oral diet should consider predominantly the issue of appropriate protein-calorie content and texture, in order to cope with nutritional deficiencies and swallowing difficulties. In addition, any practical suggestions for managing the common symptoms related to cancer treatments, leading to impaired food intake or malabsorption should be included, as well.

When dietary measures fail to meet patients' protein-calorie requirements as detected by nutritional monitoring, the prescription of energy-dense ONS should be considered, due to their proven efficacy in increasing protein-calorie intake $(6,21)$.

Complementary therapies in the form of "natural" dietary supplements are frequently used and asked for by cancer patients. Their purported antitumor effects are not yet demonstrated by appropriate efficacy evaluations, so their use cannot be recommended. However, healthcare professionals involved in the nutritional treatment of cancer patients should be knowledgeable on this issue, in order to discuss with the patients the potential risks, benefits and expectations deriving from specific dietary supplement consumption (22).

A healthy dietary pattern is known to be associated with reduced cancer risk (23), so it is reasonable to argue that it would reduce cancer recurrence, as well. However, the available clinical supporting evidence is limited to reduction of fat intake in women with early-stage breast cancer (24). Since cancer and related treatments may be responsible for metabolic changes affecting nutritional requirements, dietary advice should be tailored to the individual patient and "hypocaloric alternative anti-cancer diets" (e.g. macrobiotic or vegan) are not recommended, as they could worsen protein-calorie intake with no proven benefits on recurrence rates (25).

Finally, although recent animal model studies showed that pretreatment short-term starvation could improve chemotherapy (CT) efficacy and reduce toxicity by diminishing malignant cells' resistance to drugs while protecting normal tissues (26), this hypothesis still needs to be confirmed in humans. Therefore, this practice is not recommended, particularly in malnourished patients and those at nutritional risk, since weight and lean body mass loss is associated with dose-limiting toxicity and mortality in patients undergoing $\mathrm{CT}(3,10)$.

\section{Enteral Nutrition}

EN by means of tube feeding offers the possibility of increasing or ensuring nutrient intake whenever the gastrointestinal tract is functional and oral nutrition is not feasible or remains inadequate despite nutritional counseling and ONS consumption $(6,7,27)$.

EN should not be used routinely during anticancer treatment in all patients, but only in those who are malnourished or judged to be unable to eat adequately (the intention being to introduce an amount of calories $\geq 60 \%$ of estimated requirements) for more than 7 days $(6,7,27)$.

Tube feeding can either be delivered via trans-nasal (nasogastric / nasojejunal tube) or a percutaneous route (percutaneous endoscopic / radiologically inserted / surgical gastrostomy or jejunostomy). To date, there is insufficient evidence to recommend the best route in terms of efficacy and safety $(27,28)$; however, gastrostomy should be preferred for long term treatment (i.e. home artificial nutrition, HAN), as it may be more comfortable for patients and 
easier to manage for care-givers, whereas trans-nasal tubes need to be replaced approximately every 6 weeks $(6,7)$. Whenever trans-nasal tubes or gastrostomy placement is not feasible, as may be the case in severe obstructing esophageal or gastric cancer, needle catheter jejunostomy represents the most appropriate EN delivery route $(6,7,27)$.

With regards to timing, prophylactic feeding does not seem to offer advantages in terms of nutritional outcomes, treatment interruptions and survival compared to reactive feeding, which is initiated once nutritional counseling and ONS have failed to satisfy energy requirements $(27,28)$.

EN represents the first-line peri-operative nutritional treatment also for surgical cancer patients requiring artificial nutrition $(6,7)$. Both European and American guidelines recommend preoperative EN with immune-enhancing formulas, containing arginine, $\omega-3$ fatty acids and nucleotides, in cancer patients undergoing major head-neck or abdominal surgery $(6,7,16)$, although the grade of this recommendation is still being debated. Post-operative EN is recommended in surgical patients malnourished at the time of intervention, in those who cannot reinitiate oral nutrition early or when this is expected to be inadequate for more than 10 days (16).

\section{Parenteral Nutrition}

The use of PN in cancer patients has been debated because of the risk of infection. Both European and American guidelines clearly stated that PN is indicated in patients receiving active cancer treatment who are malnourished or are facing a period longer than 7 days of inadequate energy intake when nutritional counseling, ONS or EN are not feasible or ineffective $(4,7)$.

Routine PN during cancer treatment is strongly not recommended $(4,7)$.

A short period of PN (10-15 days) is indicated in patients with acute and severe mucositis, ileus or intractable vomiting, whereas long-term PN (more than 30 days) should be implemented in patients with intestinal failure due to extensive bowel resection, severe malabsorption, mechanical bowel obstruction, in sub-acute or chronic radiation enteritis and in patients with graft versus host disease of the digestive tract (4, 7). PN may also aid insufficient oral intake in hypophagic patients with a working gut (supplemental PN) (27).

PN is contraindicated in hemodynamically unstable patients, with ascites, severe organ failure, or in the presence of severe glycemic instability and it is rarely appropriate in incurable cancer patients with life expectancy shorter than 3 months, Karnowfsky score $\leq 50$ or ECOG performance status $\geq 3(4,7,15)$.
For long-term PN, a tunneled-catheter or implanted chamber is needed. Peripherally inserted central catheters (PICC) can also be used.

Rigorous monitoring, particularly of glycemia and electrolytes, should be implemented from the time of starting $\mathrm{PN}$, in order to prevent clinical and metabolic complications and to evaluate the impact of $\mathrm{PN}$ on clinical outcomes.

\section{Home artificial nutrition and palliative care}

Home Artificial Nutrition (HAN) is a well established extra-hospital therapy, which helps to decrease the costs of health care, mostly by reducing the number and length of hospitalizations (29). HAN can improve the prognosis of patients in several acute and chronic diseases, including cancer, and allows patients to integrate into their families and into society, thus improving their quality of life (30). Due to its organizational complexity, potentially serious complications and the necessity of periodic outcomes assessment, HAN should be prescribed and regularly monitored using defined protocols shared between oncologists and clinical nutrition specialists.

Nutritional support, including HAN, may be also integrated into palliative care programs, when it is expected to be beneficial to quality of life and if it is estimated that patients may die from malnutrition prior to dying from cancer progression $(4,27)$.

While patients who are not in the terminal phase of cancer may benefit from nutritional support and other medical therapies for cancer cachexia (31), low-quality evidence (i.e. in the absence of randomized trials) suggests that the administration of EN or $\mathrm{PN}$ in the last weeks of life does not change the course of the disease, so it may not be indicated (32).

According to international guidelines, artificial nutrition may not be appropriate in incurable cancer patients with life expectancy shorter than 3 months or Karnowfsky score $\leq 50$ or ECOG performance status $\geq$ $3(4,6,7,15)$.

In conclusion, nutritional support, including HAN, may be integrated into palliative care programs, according to individual-based evaluations, quality of life implications, life expectancy and patients' awareness.

\section{Discussion}

It should be emphasized that malnutrition is an important issue in cancer patients, which should be appropriately managed by structured collaboration between oncologists and clinical nutrition specialists.

The AIOM and SINPE recommend validated nutritional screening upon diagnosis and at regular time points in all patients with cancer type, stage or 
treatment potentially affecting nutritional status, together with prompt referral to clinical nutrition services or medical personnel with documented skills in clinical nutrition for comprehensive nutritional assessment and support prescription.

Well-designed clinical trials are needed to improve the evidence in favour of nutritional support in different care settings for cancer patients. In addition, nutritional parameters should be considered as relevant outcomes or potential confounders in outcome assessment in clinical oncology research. A summary of the AIOM-SINPE practical recommendations is reported in Table 1.

TABLE 1. Summary of the AIOM-SINPE practical recommendations for nutritional support in cancer patients

- Nutritional screening should be performed using validated tools (NRS 2002, MUST, MST, MNA) upon diagnosis and systematically repeated at regular time points in patients with cancer type, stage or treatment potentially affecting nutritional status.

- Patients at nutritional risk should be promptly referred for comprehensive nutritional assessment and support to clinical nutrition services or medical personnel with documented skills in clinical nutrition, specifically for cancer patients.

- Nutritional support should be actively managed and targeted for each patient according to nutritional conditions, clinical status, planned treatmen and expected outcome. It should comprise nutritional counseling with the possible use of oral nutritional supplements and/or artificial nutrition (enteral nutrition, total or supplemental parenteral nutrition) according to spontaneous food intake, tolerance and effectiveness.

- Nutritional support and dietary modifications should aim to assist the maintenance or recovery of nutritional status by increasing or preserving protein and calorie intake. "Alternative hypocaloric anti-cancer diets" (e.g. macrobiotic or vegan diets) are not recommended.

- Nutritional support may be integrated into palliative care programs, according to individual-based evaluations, quality of life implications, life expectancy and patients' awareness.

- Home artificial nutrition should be prescribed and regularly monitored using defined protocols shared between oncologists and clinical nutrition specialists.

- Nutritional parameters should be considered as relevant outcomes or potential confounders in outcome assessment in clinical oncology research.

- Well-designed clinical trials are needed to improve the evidence in favour of nutritional support in different care settings for cancer patients.

\section{Acknowledgement}

The research was partially supported by Ricerca Corrente Fondazione IRCCS Policlinico San Matteo, Pavia, from the Italian Health Ministry and Ricerca Finalizzata Ministero della Salute project code RF-2011-02351315.

\section{Competing Interests}

The authors have declared that no competing interest exists.

\section{References}

1. Hébuterne $X$, Lemarié E, et al. Prevalence of malnutrition and current use of nutrition support in patients with cancer. JPEN. 2014; 38:196-204.
2. Van Cutsem E, Arends J. The causes and consequences of cancer-associated malnutrition. Eur J Oncol Nurs. 2005; 9: 51-63.

3. Lu Z, Yang L, Yu J, et al. Change of body weight and macrophage inhibitory cytokine-1 during chemotherapy in advanced gastric cancer: what is their clinical significance? PLoS One 2014; 9: e88553.

4. Bozzetti F, Arends J, Lundholm K, et al. ESPEN guidelines on parenteral nutrition: non-surgical oncology. Clin Nutr. 2009; 28: 445-54.

5. Paccagnella A, Morassutti I, Rosti G. Nutritional intervention for improving treatment tolerance in cancer patients. Curr Opin Oncol. 2011; 23:322-30.

6. Arends J, Bodoky G, Bozzetti F, et al. ESPEN guidelines on enteral nutrition: non-surgical oncology. Clin Nutr. 2006; 25: 245-259.

7. August DA, Huhmann MB; American Society for Parenteral and Enteral Nutrition (ASPEN) Board of Directors. ASPEN clinical guidelines: nutrition support therapy during adult anticancer treatment and in hematopoietic cell transplantation. JPEN. 2009; 33:472-500.

8. Spiro A, Baldwin C, Patterson A, et al. The views and practice of oncologists towards nutritional support in patients receiving chemotherapy. Br J Cancer. 2006; 95:431-4.

9 Skipper A, Ferguson M, Thompson K, et al. Nutrition screening tools: an analysis of the evidence. JPEN. 2012; 36: 292-8.

10. Prado CM, Maia YL, Ormsbee M, et al. Assessment of nutritional status in cancer - the relationship between body composition and pharmacokinetics. Anticancer Agents Med Chem. 2013; 13: 1197-203.

11. Norman K, Stobäus N, Pirlich M, et al. Bioelectrical phase angle and impedance vector analysis - Clinical relevance and applicability of impedance parameters. Clin Nutr. 2012; 31: 854-61.

12. Norman K, Stobäus N, Zocher D, et al. Cutoff percentiles of bioelectrical phase angle predict functionality, quality of life, and mortality in patients with cancer. Am J Clin Nutr. 2010; $92: 612-9$.

13. Caccialanza R, Cereda E, Klersy C, et al. Phase angle and handgrip strength are sensitive early markers of energy intake in hypophagic, non-surgical patients at nutritional risk, with contraindications to enteral nutrition. Nutrients. 2015; 7:1828-40.

14. Grundmann O, Yoon SL, Williams JJ. The value of bioelectrical impedance analysis and phase angle in the evaluation of malnutrition and quality of life in cancer patients-a comprehensive review. Eur J Clin Nutr. 2015. [Epub ahead of print]. doi:10.1038/ejen.2015.126.

15. French Speaking Society of Clinical Nutrition and Metabolism (SFNEP). Clinical nutrition guidelines of the French Speaking Society of Clinical Nutrition and Metabolism (SFNEP): Summary of recommendations for adults undergoing non-surgical anticancer treatment. Dig Liver Dis. 2014 46:667-74.

16. Weimann A, Braga M, Harsanyi L et al. ESPEN Guidelines on Enteral Nutrition: surgery including organ transplantation. Clin Nutr. 2006; 25: 224-44.

17. Baldwin C, Spiro A, Ahern R, Emery PW. Oral nutritional interventions in malnourished patients with cancer: a systematic review and meta-analysis. J Natl Cancer Inst. 2012;104:371-85.

18. Langius JA, Zandbergen MC, Eerenstein SE, van Tulder MW, Leemans CR, Kramer MH, Weijs PJ. Effect of nutritional interventions on nutritional status, quality of life and mortality in patients with head and neck cancer receiving (chemo)radiotherapy: a systematic review. Clin Nutr. 2013; 32: 671-8.

19. Valentini V, Marazzi F, Bossola M, et al. Nutritional counselling and oral nutritional supplements in head and neck cancer patients undergoing chemoradiotherapy. J Hum Nutr Diet. 2012; 25:201-8.

20. Ravasco P, Monteiro-Grillo I, Camilo M. Individualized nutrition intervention is of major benefit to colorectal cancer patients: long-term follow-up of a randomized controlled trial of nutritional therapy. Am J Clin Nutr. 2012; 96: 1346-53.

21. Hubbard GP, Elia M, Holdoway A, et al. A systematic review of compliance to oral nutritional supplements. Clin Nutr. 2012; 31:293-312.

22. Frenkel M, Abrams DI, Ladas EJ, et al. Integrating dietary supplements into cancer care. Integr Cancer Ther. 2013; 12:369-84.

23. World Cancer Research Fund/American Institute for Cancer Research. Food, Nutrition, Physical Activity and the Prevention of Cancer: a global perspective expert report. Washington, DC: AICR. 1997 and update 2007.

24. Blackburn GL, Wang KA. Dietary fat reduction and breast cancer outcome: results from the Women's Intervention Nutrition Study (WINS). Am J Clin Nutr. 2007; 86:s878-81

25. Sierpina V, Levine L, McKee J, et al. Nutrition, metabolism, and integrative approaches in cancer survivors. Semin Oncol Nurs. 2015; 31:42-52.

26. Lee C, Raffaghello L, Longo VD. Starvation, detoxification, and multidrug resistance in cancer therapy. Drug Resist Updat. 2012; 15:114-22.

27. Bozzetti F. Nutritional support of the oncology patient. Crit Rev Oncol Hematol. 2013; 87: 172-200.

28. Bossola M. Nutritional intervention in head and neck cancer patients undergoing chemoradiotherapy: a narrative review. Nutrients. 2015; 7: 265-76.

29. Santarpia L, Pagano MC, Pasanisi F, et al. Home artificial nutrition: An update seven years after the regional regulation. Clin Nutr. 2014; 33: 872-8.

30. Staun M, Pironi L, Bozzetti F, Baxter J, Forbes A, Joly F, et al. ESPEN guidelines on parenteral nutrition: home parenteral nutrition (HPN) in adult patients. Clin Nutr. 2009; 28: 467-79.

31. Bosaeus I. Nutritional support in multimodal therapy for cancer cachexia. Support Care Cancer. 2008; 16:447.

32. Dy SM. Enteral and parenteral nutrition in terminally ill cancer patients: a review of the literature. Am J Hosp Palliat Care. 2006; 23:369-77. 